\title{
PRE- AND POST-OPERATIVE MANAGEMENT OF SURGICAL CASES.*
}

By Professor E. R. FLINT, M.B., Ch.B., F.R.C.S. (Professor of Surgery in the University of Leeds.)

\section{Lecture II.}

Having dealt with general principles I want to say something about preoperative preparation of a more specific kind. It should be understood that the methods to be discussed are additional and in no way alternative for patients who are found to be in need of general rehabilitation.

\section{PRE-OPERATIVE MEDICATION.}

First a word or two about preoperative medication. It is a humane thought that patients should be rendered unaware of anything from the time of leaving their beds until well after the operation, but in practice I think at the present time we have no drug capable of doing this without introducing extra risks chiefly of a post-operative pulmonary nature. I have tried many things, particularly barbiturates, and though generally the proceeding has been very satisfactory, I think, on the whole, lung complications have been more frequent and sometimes the usual dose has been insufficient and the patient has become restless and difficult to manage. I/ 6 gr. of morphia and I/ Ioo gr. of atropine 45 minutes before operation is still the safest method and usually gives the patient a feeling of indifference to subsequent proceedings. For very nervous patients this may be supplemented by 3 grs. of a barbiturate in saline given as a rectal retention enema $\mathrm{I} \frac{1}{2}$ hours before operation. I feel sure that hyoscine increases post-operative troubles especially retention of urine.

\section{STOMACH.}

For cases of pyloric obstruction the stomach must be washed out daily before operation. The fluid used does not matter, the important point is to cleanse the stomach and keep it reasonably empty. This diminishes congestion and hypertrophy and lessens the risk of serious peritoneal soiling when the organ is opened as in making an anastomosis. The ordinary stomach tube may be used or an indwelling Jutte's tube. The latter, once the patient has got used to it, is less disturbing, and has the further advantage that fluid by mouth need not be restricted; it is simply drawn off again, having satisfied in some measure the patient's sensation of thirst.

As nourishment cannot be utilised when given by mouth fluid will have to be given parenterally and extra chloride will be required to replace what is lost in the gastric secretion.

The stomach tube is of the greatest importance in persistent post-operative vomiting and should be used early, even where an anastomosis has been made between the stomach and another organ. Here Jutte's tube is definitely superior to the ordinary stomach pump. Post-operative gastric bleeding, too, is best treated by lavage in order to keep the stomach empty and contracted. I have seen adrenaline left in the stomach after the washing do good.

In patients who are bleeding from a peptic ulcer at the time of operation blood transfusion by the continuous drip method carried on during and after operation is essential. The same applies to other than gastric cases, when preoperative transfusion has not brought the hæmoglobin content above 80 per cent.

*University of London-A Course of Two Lectures delivered at St. Bartholomew's Hospital MedicaI College on February 16th and 17th, 1939. 


\section{INTESTINE.}

It is inadvisable to give aperients to any abdominal case within 48 hours of operation. It is imperative not to do so where an operation on the intestine is impending. The colon can be emptied during this time by enemata. Resting the bowel diminishes bacterial activity, and lessens the liability to post-operative distension.

For all cases of acute intestinal obstruction the stomach should be emptied: this applies equally to patients who have, or have not, vomited; you may be sure in such cases the intestines contain large quantities of fluid, which may be vomited in during operation and cause fatal flooding of the lungs.

In early intestinal obstruction this should be enough, but in late cases I believe it is an advantage gradually to decompress the intestine. This is accomplished by $\vec{\omega}$ a Miller-Abbott's tube passed well down into the intestine and sucking off the contents. It takes a little time, but there are a few available hours, since in these cases time spent in rehydration is well worth while. Of course if the case is one of strangulation immediate operation is imperative. The same kind of treatment should be applied to paralytic ileus. More time is available here, since no operation of is required, and it is as well, because the tube may be very difficult to get beyond the stomach. It does go eventually and there is no question that emptying the intestine in these cases hastens recovery of intestinal tone. It is useless fussing the paretic gut with aperients or hypodermis drugs; rest is what is needed and is best accomplished by large doses of morphia and sucking out the contents.

\section{THE LIVER.}

Though we have, at present, no really efficient test to give us a satisfactor idea of the manner in which the liver is performing its various functions, we do kno from microscopic examination of pieces of liver removed at operation that some degree of hepatitis is very frequently present in diseases of the biliary tract, and may be very pronounced in cases of obstruction of the common bile duct by stone. The lesion takes the form of a small celled infiltration around the small bile ducts with fatty degeneration and sometimes actual necrosis of the liver cells.

It is well, therefore, in spite of the absence of functional test evidence, to prepare these patients very carefully. The measures required are mainly those already discussed under the headings of water and glucose with the addition of that directed against the tendency to hæmorrhage.

This tendency is in some way related to jaundice of the obstructive type. Jaundice is due to hyperbilirubinæmia, and generally speaking, the deeper the jaundice is and the longer it has lasted, the greater is the risk of bleeding. However, $\bigcirc$ I do not think it is the excess of bilirubin which is responsible; this is rather an indication of liver injury by backpressure, as is a high urea content of the blood indicative of kidney damage in cases of urinary obstruction. Indeed the same predisposition to bleed is seen in common duct cases where jaundice may not be deep but in which there is co-existing cholangitis, and certainly this would seem to $\widetilde{N}$ be due to serious liver injury. I think, for practical purposes it is right to say that $\omega$ high or rising values of bilirubin in the blood are likely to be associated with a risk

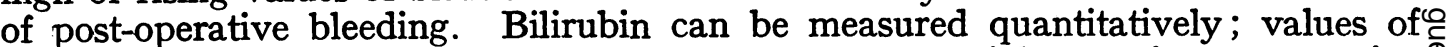
2 mgrms. per cent. or more should make one take special care in preoperative preparation and particularly if the daily estimation shows rising values.

So much so that a short waiting policy is a wise preoperative measure when the jaundice is due to stone, since in such a case it is the general rule for icterus to 
subside after a week or so. Should the jaundice begin to lessen, the risk of bleeding does so too, and almost certainly because of relief to the liver, but one should still take precautions. As well as giving water freely, which has among its beneficial effects that of improving kidney function (this is peculiarly apt to be deranged in jaundiced cases), glucose should be pushed. Clinical, as well as experimental, evidence is quite definite that insults to the liver, such as will arise at operation, are less serious when the liver is well supplied with glycogen. In this connection I should like to say that I believe spinal anæsthesia is preferable to general anæsthetics -chloroform is undoubtedly a hepatic poison, and I think ether also in a lesser degree. I always use spinal anæsthesia for my jaundiced patients.

All these measures, by improving the hepatic state, will diminish to some extent the tendency to bleed, but in addition it is proper to take certain precautions of a more specific kind indicated as follows:

I. Calcium.

2. Blood transfusion.

3. Vitamin $\mathbf{K}$.

Calcium. It is customary to inject 5 c.c. of ro per cent. calcium chloride intravenously on three or four consecutive days before operation. There is no shortage of calcium in the blood, but it is said to be immobilised by union with some toxin-immobilised, that is to say, so far as it is concerned in the clotting process. I doubt this, for it seems to me that the value of calcium, if any, is through some action on the liver, since it tends to promote recovery in animals with acute liver damage initiated by carbon tetrachloride, and I think in human beings suffering from toxic necrosis of the liver.

I should like to remind you that immediately after the injection is started the patient experiences a disagreeable sensation of heat beginning in the mouth and becoming general. This creates in the patient a feeling of anxiety, but it is not a matter of any consequence and the patient may be assured that it will pass off in a few seconds.

Blood transfusion. This, on the other hand, seems quite definitely to abolish or greatly diminish this risk of hæmorrhage, and in my opinion is the method of choice; at the same time it provides material which acts in many other ways for the good of the patient.

Transfusion of blood should be given routinely in jaundiced patients before operation. My usual practice is to give a pint or so from a suitable donor two or three days before operation, and a similar amount twenty-four hours before operation, by the tube and funnel method, having collected the blood in a graduated, warmed flask containing about 3 ozs. of 2 per cent. sodium citrate. This is, I think, by far the simplest way and does not necessitate the coming together of donor and recipient. A febrile reaction with or without rigor, may occur within 30 minutes, as it may, after any other method, even giving saline only. This is undesirable but does not seem to entail any serious consequences. What does matter is complaint of pain by the recipient during the receipt of the first few drachms of blood; this kind of reaction is typically initiated by pain in the loins, chest and abdomen and is a warning to stop the transfusion at once. It is due to incompatability of the blood, even when the preliminary grouping, which is always done, has been apparently satisfactory.

Large quantities of blood do not seem to be required in jaundiced cases as a rule, and therefore, the continuous drip method is not necessary. When a patient has bled profusely, and an operation is imperative, as in certain cases of bleeding 
peptic ulcers, then the continuous drip method preoperatively is most useful, and can be carried on through and after operation.

Vitamin K. I have no satisfactory experience with this, but in America it is quite favourably commented on, especially when given with bile salts or whole bile. It is a fat soluble vitamin found in spinach, sprouted oats, alfalfa and certain animal foods such as fish meal. It is said to raise the prothrombin level in circulating blood. The extract can be given intramuscularly.

I believe it is beneficial after operation with a tube in the common duct to collect the escaping bile and by means of a stomach tube feed it into the patient's alimentary tract. The benefit is, I think, not related to hæmorrhage, but to assimilation of food and to improvement in bowel function generally. The loss of bile means loss of most of the fat in the diet, and frequently causes considerable constipation. One practical hint I would give is, do not let the patient know what is being done, as the proceeding is apt to cause nausea from a sensation of disgust.

\section{BLOOD STATES.}

There are certain conditions associated with blood changes for which surgery is generally considered the correct treatment, such as acholuric jaundice, thrombocytopænia and splenic anæmia and one in which surgery may have to be done, though not as part of the treatment of the condition-hæmophilia. For these blood transfusion is often necessary.

In acholuric jaundice it should be given post-operatively when the spleen has been removed, otherwise the foreign blood may be quickly destroyed. If anæmiao is marked transfusion should be done before operation as well.

For thrombocytopania transfusion should be performed before operation and more than one may be necessary according to the response shown by the platelets. This is one of the conditions in which the continuous drip method may be required, especially in what is known as the acute type when the patient may be critically ill.

In splenic ancemia blood is necessary before operation when the patient has lost blood as is often the case. There is some risk here of furthering clotting in the splenic vein. Judgment is required in these cases, particularly if it is a question of giving blood after operation.

Hamophiliacs sometimes develop surgical lesions for which operation is imperative. They should have pre-operative blood transfusion, and will thereby be made safe in most instances for the duration of the operation, but more blood may be needed after operation.

\section{KIDNEYS.}

The importance in surgery of adequate elimination of waste products is obvious, and I have already stressed the necessity of giving plenty of fluid without which the kidneys cannot function properly. There is, however, another surgical aspect in the urinary tract which calls for comment as a preoperative measure-viz., relief to the kidneys in case of back pressure which is best exemplified in prostatic obstruction. The pressure at which fluid is excreted by the glomeruli is about 20 per cent. less than the carotid pressure, i.e. about Ioo $\mathrm{mm}$. Hg. The proteins in the blood exert an osmotic pressure of some $25 \mathrm{~mm}$. Hg., so actually the filtering force in the glomeruli is $75 \mathrm{~mm}$. $\mathrm{Hg}$., and can only proceed when the ureteric pressure is below this figure. Prostatic obstruction raises the pressure in the ureters, and gradually exerts a restraining 
influence on filtration causing waste products to accumulate in the blood, and injury to the kidneys which may be enhanced by coexisting infection. A rise in blood urea in these cases is taken as an indication of deficient kidney function, which must be improved before any radical operation is performed. In this respect relief to back pressure by an indwelling catheter or suprapubic cystostomy may be properly regarded as a preoperative measure. For this reason the twostage removal of the prostate has become a procedure well recognised as making for safety. If a patient has retention with distended urinary bladder, gradual decompression by catheter should be practised, and when it has thus been overcome suprapubic cystostomy should be done as a preliminary to prostatectomy.

The danger of suddenly relieving pressure on the kidneys is well known; it may induce acute suppression since sudden relief to the kidney tubules releases pressure on the vascular elements, and the kidneys become suddenly engorged reestablishing pressure of another kind, and of a higher degree than before.

There is a principle here which applies to other parts of the body e.g. sudden relief to back pressure on the liver in obstruction of the common duct; in acute intestinal obstruction, and in compression of the brain.

So far as the distended bladder is concerned it is easy to reduce pressure gradually; it is not so easy in the other instances, but can be done by exercising a little ingenuity. I have mentioned Jutte's tube and Miller-Abbott's tube in connection with the intestine. In common bile duct obstruction e.g. by malignant disease of the head of the pancreas, gradual decompression is also possible by drawing off at first during operation, a little bile by aspiration from the gall bladder and then anastomosing it to the stomach in the following way. The peritoneal suture is first applied to approximate the organs, then the mucosal layer is coagulated on both organs by diathermy without opening the lumen of either viscus; a small puncture is made through the coagulated line on both sides and these small openings brought together by the mucosal stitch, and finally the peritoneal suture completed. The effect is that after releasing the clamps the gall bladder slowly empties into the stomach through the small puncture, and presently the rest of the mucosal line sloughs and opens up the whole of the line of anastomosis. I have done this several times with very satisfactory results. Perhaps one cannot call this preoperative preparation, but it serves to illustrate the application of an important principle, since sudden relief to the liver, as to the kidney, causes acute congestion of the organ, and may be followed by what is known as liver deathquite comparable to the uræmic death in the case of the kidney.

\section{THYROID.}

In the condition known as hyperthyroidism, whether primary or secondary, there is excessive secretion, possibly an altered secretion, by the hyperplastic gland associated with an increased metabolism throughout the body. Experience has taught us that to operate in this condition without careful preparation is inviting disaster. Not that there is any difficulty in partial or complete thyroidectomy from the surgical point of view, but the operation will almost certainly exaggerate. the previous symptoms precipitating the patient into a state of thyrotoxic crisis, in which the already overdriven heart is unable to bear the extra strain, passing into a state of auricular fibrillation. In these circumstances the death rate is high, (it used to be around 50 per cent.), after preoperative preparation the mortality is reduced to somewhere about 3 per cent. This is a very obvious instance of the triumph of preparing patients for operation. 
There are several factors in the preparation of these patients. They are frequently dehydrated from one cause or another, and therefore need extra fluid; since vomiting may be a prominent symptom the fluid may have to be given parenterally. Glucose, too, is necessary for the liver is deprived of its glycogen and there may be jaundice; indeed in some of the fatal cases the symptoms are indistinguishable from those in the so-called liver deaths. Many thyrotoxic patients have glycosuria, but this bears no relation to the carbohydrate intake, and is no contra-indication to generous quantities of glucose in the diet. Insulin may be very useful, though its effects should be closely watched, since the treatment of a possibly induced hypoglycæmia is not easy when the liver is devoid of glycogen.

\section{If heart failure is impending complete digitalisation should be established.}

Mental irritability should be treated with luminal or bromides, but the most important factors in preparation are rest and iodine. Prolonged rest in bed free from all disturbing elements will do a lot towards restoring heart reserve, and it is well to begin with this early so as to bring its maximum effect into the period of treatment with iodine. It is generally agreed that iodine therapy should be concentrated into the fourteen days prior to operation, since its effect is apt to wane after this and it is rarely so efficient in a second course. It is usually given as Lugol's solution ( 5 per cent. iodine in Io per cent. potassium iodide) in doses of 5 to Io minims t.d.s. In cases exhibiting an undue reaction after operation iodine should be continued and glucose pushed. In the really severe post-operative reactions Lugol's solution may be given intravenously in doses of 15 minims.

The effect of iodine is very striking both upon the general condition of the patient, and in lessening vascularity of the thyroid gland. Clinically the pulse rate may drop from I 20 or more to 80 or 90 per minute and the metabolic rate? from 50 per cent. or even roo per cent. above normal in bad cases, to 20 per cent. or 30 per cent. above normal and the patient begins to put on weight. These are the chief criteria by which one judges the improvement in the patient.

I should like to mention here, though it has no connection with hyperthyroidism, that giving thyroid extract together with dieting is good preoperative treatment for very fat patients. Since many gall stone cases are very fat this applies particularly to them and since the operation is often not a matter of urgency many weeks may advantageously be spent in this effort. Obese patients, generally speaking, run an extra operative risk by reason of their excessive stoutness.

\section{DIABETES.}

Certain conditions are very prone to occur in diabetics, viz., carbuncle, cellulitis and gangrene, and, of course, a diabetic may develop any of the ordinary surgical lesions, though no more apt to do so than the non-diabetic. In either case careful preparation is necessary. When there is time the blood sugar should be reduced to normal by diet and regulated insulin before operation is undertaken. Fluid should be given practically up to the last, and 2 ozs. of sugar with I5 to 20 units of insulin two hours before operation.

Since diabetics are frequently dehydrated and very liable to ketosis, local or spinal anæsthetics are less prone to induce either of these dangers than ether and especially chloroform. For several hours after operation the same principles should obtain and progress checked by blood examinations for sugar. Larger doses of insulin will be needed when infection is present, since this reduces its efficiency. 
The outlook for properly prepared diabetics is good, but in assessing prognosis one must remember that vascular changes are one of the effects of diabetes, and such lesions may have a material influence on post-operative behaviour of the patient.

There are many. less common diseases and others in the more specialised branches of surgery, for which specific methods of preparation are required but there is no time to deal with them. Moreover I have purposely confined my remarks to those conditions with which general surgeons are concerned.

I have, too, concentrated on preoperative preparation as the post-operative period is governed very largely by what is, or is not done, before and at operation.

\section{POST-OPERATIVE TREATMENT.}

Should there be trouble after operation many of the measures we have been considering are equally applicable at this stage and for similar reasons. There are, however, one or two additional remarks I would like to make.

After a straightforward abdominal operation there is only one distressing feature as a rule during convalescence and that is flatulence.

It is not worrying to the surgeon but is very uncomfortable to the patient and usually passes off in a day or two. The best way of dealing with it is by morphia and occasional enemata. There is no doubt it arises through disordered peristaltic rhythm from the manipulations at operation, and intestinal rest is the quickest way to rectify the trouble.

Another post-operative complication is peritonitis. This should be preventable, but still accounts for many deaths, even in cases not infected at the time of operation. Care at operation is the solution of most cases, but I have no doubt in a patient of poor risk slight soiling may lead to serious trouble, whereas it would have no ill consequences in the ordinary type of case. Careful preparation, therefore, will tend to prevent this complication. Attempts have been made to increase the resistance of the peritoneum against infection by intraperitoneal injections of substances which promote a high cell count in the peritoneal fluid. Nucleolates have been used, but now it is more usual to inject a vaccine produced from a mixture of streptococci and colon bacilli obtained from patients who have died of peritonitis. It is introduced into the peritoneal cavity by puncture some 3 days before operation, as the reaction seems to reach its maximum in this time. The dose is 0.5 to I c.c. containing some $500,000,000$ organisms. The type of case for which it is most beneficial is resection of the colon in which it is difficult to avoid peritoneal soiling entirely.

When post-operative peritonitis has occurred the patient should be put in the Fowler position-more upright than the usual semi-sitting posture adopted after abdominal operations routinely-and the stomach kept empty by an indwelling Jutte's tube, fluid administered parenterally, and distension relieved by enemata. Large and frequent doses of morphia are required to ensure complete rest of the intestines which is so important. Fomentations are useful, and sulphonamide should be given intramuscularly. Aperients, pituitrin and eserine are to be avoided at first.

\section{Lungs.}

With modern methods of anæsthesia post-operative lung complications are less frequent than they used to be, but are still unpleasantly common. Waterlogging 
accounts for some of them, and more often than not, is an indication of peripheral circulatory failure for which preoperative preparation is the best preventive. I think, perhaps, we might do something more in the preoperative stage by making use of certain physiological techniques to determine functional efficiency and to improve vital capacity, perhaps by deep breathing. According to Y. Henderson (I93I) the circulatory volume depends not only on the heart and vasomotor system, but on a third factor - the veno-pressor mechanism, which is mainly determined by general tonus of skeletal muscle, more especially of the abdominal muscles and particularly the diaphragm. In general the degree of activity of respiration, the general tonus of the body and the volume of the circulation vary together. Accordingly it is found that in the physical depression after major operations certain conditions occur simultaneously-the diaphragm is relaxed, the tonus of the skeletal muscles and of the alimentary canal is lowered, and the volume of the circulation diminished. Under carbon dioxide inhalation all these aspects of depression improve together. Since many post-operative lung conditions originate in atelectatic areas hyperventilation should do a great deal as a preventive.

Towards the end of an operation my patients are made to breathe ro per cent. carbon dioxide with oxygen for short periods, and this is continued in bed for several hours. The conscious patient will show evidence of distress if this mixture is breathed for more than a few minutes; in the unconscious patient definite harm may result. It is therefore important to make the periods short; in fact it is unnecessary that more than half a dozen deep breaths should be taken if the performance be frequently repeated.

On a few occasions I have asked a bronchoscopist colleague to assist; he has succeeded in removing a thick tenacious lump of mucus from a bronchus and the instant relief has been dramatic. From being apparently a very ill patient, blue, dyspnœic, apprehensive, with tachycardia and ineffective cough he becomes at once a good colour and calm with a rapidly falling pulse rate and expresses himself as feeling all right.

\section{Heart.}

I have said little about the heart since in the absence of obvious cardiac disease it is rarely a major factor in post-operative conditions primarily. When it becomes exhausted it is generally because of some pre-existing condition such as circulatory failure at the periphery, with which I have already dealt.

\section{CONCLUSION.}

I hope I have made it clear that in the debilitated, dehydrated patient, however skilful an operator may be, it is not enough for this type of case. It is a trite saying that prevention is better than cure; it was never more true than in relation to these matters. Undoubtedly to the patient the crucial event is the operation; to the surgeon the important question to be answered, in order to ensure the safety of his patient, is often when, rather than how, to operate. The answer to this question, if it is not to be a mere guess, involves a knowledge of things which carries him far beyond the borders of mere operative technique. 